\title{
Study on Heavy-Loaded Locomotive Schedule of Da-qin Railway
}

\author{
Ruiye Su, a , Leishan Zhou ${ }^{2, b}$ and Jinjin Tang ${ }^{3, c^{*}}$ \\ ${ }^{1}$ School of Traffic and Transportation, Beijing Jiaotong University, Beijing 100044, China \\ ${ }^{2}$ School of Traffic and Transportation, Beijing Jiaotong University, Beijing 100044, China \\ ${ }^{3}$ School of Traffic and Transportation, Beijing Jiaotong University, Beijing 100044, China \\ a suruiye@bjtu.edu.cn, ${ }^{b}$ Ishzhou@bjtu.edu.cn, ${ }^{c}$ jjtang@bjtu.edu.cn
}

Keywords: Locomotive Schedule; Heavy-loaded Transportation; Da-qin Railway; Optimization

Abstract: The main difference between locomotive schedule of heavy-loaded railway and that of regular rail transport is the number of locomotives utilized for one train. One heavy-loaded train usually has more than one locomotive, but a regular train just has one locomotive. Based on analysis of the current locomotive schedule in Da-qin Railway, this paper develops an optimization model for multi-locomotives scheduling problem. The objective function of the proposed model is the minimization of total number of utilized locomotives. The optimization model for multi-locomotives scheduling is the non-deterministic polynomial hard problem. Therefore, we convert multi-locomotive traction problem into single-locomotive traction problem. Then, the single-locomotive traction problem can be converted into assignment problem. Hungarian algorithm is the one of best choices to solve the model and obtain the locomotive schedule. It can significantly reduce the number of locomotives used. It uses the variance of detention time of locomotives at stations to evaluate the stability of locomotive schedule. In order to evaluate the effectiveness of the presented optimization model, case studies are conducted which implements the proposed model for generating locomotive schedules for 20kt heavy-loaded combined trains and 30kt heavy-loaded combined trains on Da-qin Railway, respectively. Results of case studies show that the proposed model could save 13 HXD locomotives and 49 SS4 locomotives for the locomotive schedule of operating 20kt heavy-loaded combined trains and it can save 47 SS4 locomotives and the number of HXD locomotives can also meet the needs for the locomotive schedule of operating $30 \mathrm{kt}$ heavy-loaded combined trains, compared with the current locomotive schedule. Therefore, the effectiveness of the proposed optimization model and its corresponding solving method is validated by case studies.

\section{Introduction}

Da-qin Railway is the earliest double-track electrified railway in China which carries 10kt and 20kt heavy-loaded trains. As shown in Fig. 1, with the rapid development of the economy, the coal demand in China increases every year. Although, there was a slight decrease of the coal demand in 2014, it still reaches to 3.59 billion tons. It can be found that the freight volume of Da-qin Railway was more than 200 million tons in 2005, which already reached the theoretical limit of international heavy-loaded railway transport. Moreover, it increased to 400 million tons in 2010 and 450 million tons in 2014. However, the transported cargo volume still cannot meet the Chinese coal demand. It still needs to improve freight volume. It is difficult for current train schedule to increase the freight flow density and train's speed in Da-qin Railway, the method to increase freight volume is that increase of transportation capacity of a train. Therefore, it needs to operate 30kt combined trains in Da-qin Railroad. 


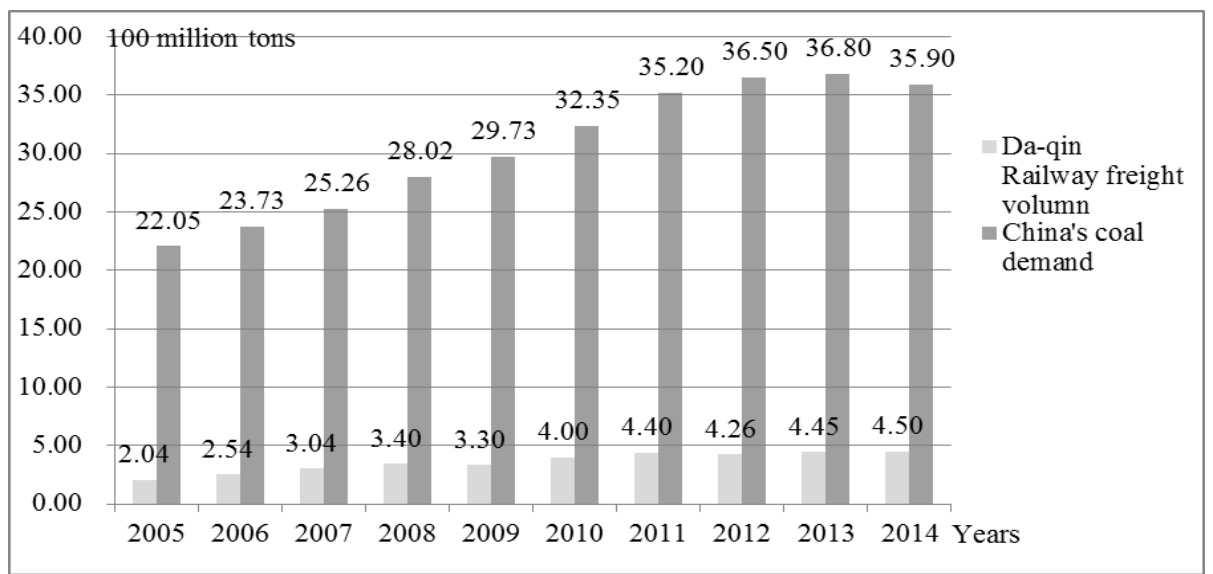

Fig. 1. China's coal demand and Da-qin Railway freight volume from 2005 to 2014 [1]

Locomotives are a critical factor for the capacity of a railroad. The effectiveness of locomotive scheduling has a directly impact on the efficiency the operation cost, and benefits of a railway. Therefore, a study for efficiency and equilibrium of the locomotive scheduling in Da-qin Railway is necessary for operating $30 \mathrm{kt}$ combined trains.

Some researches of heavy-loaded locomotives mainly focus on the technical level that concentrates on researching basic technologies. Cheng et al [2] provided technical support for the application of Reliability Centered Maintenance (RCM) in railway locomotive and verified its effectiveness in keeping reliability and reducing maintenance cost. Zheng and Wei [3], Chang [4] and $\mathrm{Mu}$ [5] analyzed the mechanical response of railway track structure under axle loads from 25t to 40t. Raviv and Kaspi [6] and Kumar and Bierlaire [7] proposed a mixed-integer linear program model that determines the optimal strategy. There are few optimization researches for locomotive schedule, because the small density of heavy-loaded trains, short length of station track, and the difficult transport organization. Meanwhile, these researches results of heavy-loaded locomotives mainly relate to 10kt 20kt heavy-loaded trains. On the other hand, some researches focus on the following areas: 1) Ji et al [8] used computers to achieve double-locomotive traction control and determine locomotive marshalling schedule; 2) Ruppert et al [9] analyzed how to increase the usage efficiency and reliability of locomotives; 3) Kuo and Nicholls [10] developed a mixed integer linear program (MILP) to determine the least-cost plan of allocating locomotives and improve locomotive utilization; 4) Aronsson, Kreuger and Gjerdrum [11] built a MIP model to solve locomotive routing and scheduling problem and Vaidyanathan, Ahuja and Orlin [12] formulated the locomotive routing problem (LRP) as an integer programming problem on a suitably constructed space-time network and developed robust optimization methods to solve it; 5) Ghoseiri and Ghannadpour [13], Kasalica, Mandic and Vukadinovic [14], Rouillon, Desaulniers and Soumis [15] and Teichmann et al [16] were devoted to the research of locomotive assignment problem, and used different methods to optimize it; and 6) Li et al [17] established a mathematical model for making a locomotive working diagram of a train diagram with paired trains and used the improved ant colony algorithm to solve it. Most of researches results of locomotive schedule relate to the regular trains, and only a few of them involve 10 20kt heavy-loaded trains. There are no researches of locomotive scheduling for 30kt heavy-loaded trains.

\section{Criteria of Locomotive Schedule}

The criteria of locomotive schedule can reflect the work quality, efficiency and benefit of locomotives, which include quantitative criteria, quality criteria and equilibrium criteria. The major performance index of quantitative criteria is the number of available locomotives. The most critical performance measure of quality criteria is coefficient of locomotive requirement. The most important performance factor of equilibrium criteria is the equilibrium degree of locomotive schedule.

Number of Available Locomotives. Number of available locomotives is the total number of locomotives which are used in the whole turnaround process [18]. The less number is, the higher 
efficiency will be. The general method to determine the number of available locomotives are graphic method and direct calculation method. The graphic method is based on locomotive schedule to determine the number of used locomotives. And the formula of direct calculation method is listed below:

$$
M_{f}=\frac{\left(n+n_{d}+n_{t}\right) g_{l}}{24}=u_{s} K_{d} .
$$

Where $M_{f}$ is the number of available locomotives in each traction section; $n$ is the total number of trains; $n_{d}$ is the number of trains with double-locomotive traction; $n_{t}$ is the number of trains with three-locomotives traction; $\theta_{l}$ is the locomotive complete turnaround time; $\mu_{s}$ is the number of supplied locomotives; $K_{d}$ is the coefficient of locomotive requirement in traction sections.

Coefficient of Locomotive Requirement. Coefficient of locomotive requirement indicates the number of locomotives required per one pair of trains in a traction section [18]. One locomotive completes a turnaround means it completed a traction task. The coefficient of locomotive requirement can be calculated by the equation below:

$$
K_{d}=\frac{\theta_{l}}{24} \text {. }
$$

Equilibrium Degree of Locomotive Schedule. In addition to quantitative criteria and quality criteria, the equilibrium criteria are also very important in evaluation criteria of locomotive schedule, which refer to the difference between detention time of each locomotive at stations and running time of each locomotive. At the same section, the running time is the same. Therefore, in the actual evaluation process, the variance of detention time of each locomotive at stations, $D$ (equilibrium degree), can be used to describe the equilibrium of locomotive schedule. It can be calculated as below:

$$
D=\frac{1}{n} \sum_{i=1}^{n}\left(\mathrm{c}_{i}-\mathrm{E}\right)^{2} \text {. }
$$

Where $n$ is the total number of locomotives which undertake traction tasks; $i$ is a locomotive that undertakes traction tasks, i.e. $i \in n ; c_{i}$ is the detention time of a locomotive at stations; $E$ is the average detention time of locomotives at stations.

Equation (3) shows that equilibrium degree $D$ which refers to the discrete degree between $c_{i}(i=1,2$, $3, \ldots, n)$ and $E$. The smaller the value of $D$ indicates the smaller the difference and the more balance the locomotive schedule.

\section{Model Description}

Some assumptions are presented in this model: 1) the train schedule is known and invariant in a certain period of time, i.e. the impact of train delay will be ignored; 2) based on the line length of Da-qin Railway $(653 \mathrm{~km})$, locomotive routing and locomotive running time (e.g. a round-trip time more than 17h), it assumes within 24 hours all available locomotives (the total locomotives after deducting the locomotives in maintenance and preparation) are without maintenance; 3 ) for the trains with multi-locomotive traction, locomotive reconnection between HXD locomotives and SS4 locomotives is not be considered. And the time of locomotive reconnection and dismantling was included in the detention time of locomotives at stations; 4) in a certain period of time, the locomotive crew working system in Da-qin Railway is invariant, so the effects of locomotive crew working system and locomotive crew shift mode on locomotive scheduling are not considered; and 5) in the daily initial time (18:00), each station has enough locomotives which at least are equal to the number of locomotives in need, i.e. the locomotive homing will not be considered.

Notations and Sets. In the process of constructing the optimization model of heavy-loaded locomotive schedule, there are some notations and sets will be used:

$K$ is the set includes all of stations on the railway;

$k$ is a station on the railway, i.e. $k \in K$; 
$N_{k, d}$ is the total number of trains arriving at station $k$;

$N_{k, f}$ is the total number of trains departing from station $k$;

$i$ is the locomotive arriving at station $k$, and $i=1,2,3, \ldots, N_{k, d}$;

$j$ is the train departing from station $k$, and $j=1,2,3, \ldots, N_{k, f}$;

$T_{k}$ is the standard detention time of locomotives at station $k$;

$k_{i, f}$ is the departure station of locomotive $i$ is station $k$;

$t_{i, f, k}$ is the departure time of locomotive $i$ at station $k$;

$k_{i, f}$ is the destination station of locomotive $i$ is station $k$;

$t_{i, d, k}$ is the arrival time of locomotive $i$ at station $k$;

$k_{j, f}$ is the departure station of train $j$ is station $k$;

$t_{j, f, k}$ is the departure time of train $j$ at station $k$;

$k_{j, d}$ is the destination station of train $j$ is station $k$;

$t_{j, d, k}$ is the arrival time of train $j$ at station $k$.

Parameters. In the process of developing the optimization model, the known parameter that will be involved is $c_{i, j}^{k}$. It refers to the waiting time which is equal to the detention time of locomotive $i$ at station $k$ minus the standard detention time. The waiting time $c_{i, j}^{k}$ of locomotives at stations can be calculated by

$$
c_{i, j}^{k}=\left\{\begin{array}{lc}
t_{j, f, k}-t_{i, d, k}-T_{k} & t_{j, f, k}-t_{i, d, k} \geq 0 \text { and } k_{i, d}=k_{j, f} \\
t_{j, f, k}-t_{i, d, k}-T_{k}+1440 & t_{j, f, k}-t_{i, d, k}<0 \text { and } k_{i, d}=k_{j, f} . \\
\infty & k_{i, d} \neq k_{j, f}
\end{array}\right.
$$

Variable. The variable is assumed to be $c_{i, j}^{k}$, which refers to whether locomotive $i$ hauls train $j$ or not at station $k$. The value of it is

$$
x_{i, j}^{k}=\left\{\begin{array}{rr}
1 & \text { locomotive } i \text { hauls train } j \\
0 & \text { locomotive } i \text { doesn't haul train } j
\end{array} .\right.
$$

Objective Function. The primary aim of locomotive scheduling is to complete planed traction tasks. Based on that, the less number of locomotives are, the lower transportation cost will be. Therefore, the objective function of heavy-loaded locomotive scheduling problem is that minimizing number of locomotives, that is

$$
\min Z^{\prime}=\frac{T_{t}+T}{1440} \text {. }
$$

Where $T_{t}$ is the total time of trains running from departure station to destination station; $T$ is the total detention time of locomotives at stations, which can be calculated by

$$
T=T_{K}+T_{w} .
$$

Where $T_{K}$ is the total standard detention time of locomotives at stations; $T_{w}$ is the total waiting time of locomotives at stations.

For the established train schedule, the $T_{t}$ is a constant. So the minimum number of locomotives refers to the minimum detention time $T$ of locomotives at stations. Meanwhile, the $T_{K}$ is also a constant. So the minimum detention time of locomotives at stations refers to the minimum waiting time $T_{w}$ of locomotives at stations. That is

$$
\min Z=T_{w}=\sum_{k=1}^{K} \sum_{i=1}^{N_{k, d}} \sum_{j=1}^{N_{k, f}} c_{i, j}^{k} x_{i, j}^{k} .
$$


Then the equilibrium degree $D$ of locomotive schedule can be calculated by

$$
D=\frac{1}{N_{k, d}+N_{k, f}} \sum_{k=1}^{K} \sum_{i=1}^{N_{k, d}} \sum_{j=1}^{N_{k, f}}\left(c_{i, j}^{k} x_{i, j}^{k}-\frac{Z}{N_{k, d}+N_{k, f}}\right)^{2} .
$$

Constraints. The constraints are listed below:

(1) Traction locomotives constraint

$$
\sum_{i=1}^{N_{k, d}} x_{i, j}^{k}=h \quad \forall j \in N_{k, d}, \quad \forall k \in K .
$$

This constraint shows that the number of traction locomotives for each train is $h$. The value is shown in Table 1.

\begin{tabular}{cccc}
\hline $\begin{array}{c}\text { Types of } \\
\text { heavy-loaded } \\
\text { trains }\end{array}$ & $\begin{array}{c}\text { Reference } \\
\text { value }\end{array}$ & $\begin{array}{c}\text { Types of } \\
\text { heavy-loaded } \\
\text { trains }\end{array}$ & $\begin{array}{c}\text { Reference } \\
\text { value }\end{array}$ \\
\hline $\begin{array}{c}\text { 10kt heavy-loaded } \\
\text { combined trains }\end{array}$ & 2 & $\begin{array}{c}\text { 20kt heavy-loaded } \\
\text { combined trains }\end{array}$ & 2 \\
$\begin{array}{c}\text { 10kt heavy-loaded } \\
\text { unit trains }\end{array}$ & 1 & $\begin{array}{c}\text { 30kt heavy-loaded } \\
\text { combined trains }\end{array}$ & 3 \\
$\begin{array}{c}\text { 15kt heavy-loaded } \\
\text { combined trains }\end{array}$ & 2 & - & - \\
\hline
\end{tabular}

(2) Traction tasks constraint

Table 1. The value table of parameter $h$

$$
\sum_{j=1}^{N_{k, f}} x_{i, j}^{k}=1 \quad \forall i \in N_{k, f}, \quad \forall k \in K .
$$

This constraint shows that each locomotive can haul one train at the same time only.

\section{Transformation of Heavy-Loaded Locomotive Scheduling Problem}

First of all, the locomotive scheduling problem needs to be transformed into an Assignment Problem. And then the optimization results are obtained by using Hungarian algorithm.

For an unpaired train schedule, the number of arrival trains is not equal to the number of departure trains at some stations. So the unpaired train schedule needs to be transformed into a paired train schedule before solving the locomotive scheduling problem. For the paired train schedule, the number of arrival trains is equal to the number of departure trains at each station. Therefore, if each train is hauled by the same type of locomotive, the locomotive scheduling problem can be transformed into a standard Assignment Problem by treating arrival locomotives as "personnel" and departure trains as "missions".

Therefore, the transformation of heavy-loaded locomotive scheduling problem mainly focuses on the following three aspects:

(1) Transforming unpaired train schedule into paired train schedule

Usually, the train will be operated smoothly according to the train schedule. Because the number of trains in train schedule is certain on the known railway, the number of trains within $24 \mathrm{~h}$ is determined. If the train schedule is unpaired, it needs to add some "virtual operation curves". Then the number of arrival trains is equal to the number of departure trains in each section. It assumes that the number of "virtual operation curves" is $Y$ and the number of actual trains is $J$.

(2) Transforming multi-locomotive traction into single-locomotive traction

If the total number of trains is $Y+J$; the number of train with double-locomotive traction is $W$; and the number of train with three-locomotives traction is $V(W+V \leq J+Y)$. Then the number of "virtual trains" is $W+2 V$ when multi-locomotive traction is transformed into single-locomotive traction. At 
the same time, it needs to ensure that the information of train schedule is consistent with before. And then trains will be sorted and coded according to the order of departure time, and the code of trains may be $1,2,3, \ldots, J+Y+W+2 V-2, J+Y+W+2 V-1, J+Y+W+2 V$.

(3) Transformation between different locomotive types

There are two locomotive types which include SS4 locomotive and HXD locomotive in Da-qin Railway. 10kt heavy-loaded combined trains are hauled by SS4 locomotives while 10kt heavy-loaded unit trains, 15kt heavy-loaded combined trains and 20kt heavy-loaded combined trains are hauled by HXD locomotives. There is not locomotive reconnection between HXD locomotives and SS4 locomotives. Therefore, locomotive scheduling problem which is involved in two locomotive types can be considered separately for each type in order to obtain the optimization solutions. Then, the optimization objective of the locomotive schedule on the whole railway is achieved.

\section{Case Study}

Evaluating the Feasibility of Heavy-Loaded Locomotive Schedule Model. Before using the model and algorithm to solve actual problems, it needs to ensure that they are feasible. This is the content of this section.

\section{Data source and processing}

As shown in Fig. 2, line A-B-C-D is a double-track railway (section A-D for short) which is specially used to transport coal. Station B is the station where locomotive depot is located, and the standard detention time of locomotives at stations is $125 \mathrm{~min}$. Station A, station C and station D are the station where locomotive turnaround depot is located, and the standard detention time of locomotives at stations is $50 \mathrm{~min}$.

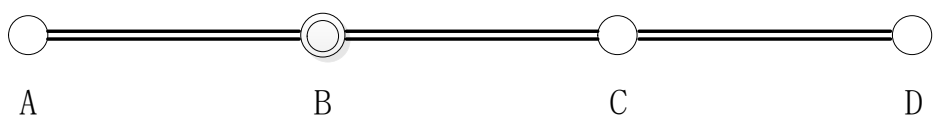

Fig. 2. Network of hypothetical railway line

There are 8 trains ( 4 pairs) which include 2 trains ( 1 pair) in section A-B, 4 trains ( 2 pairs) in section B-C and 2 trains (1 pair) in section B-D on the railway. If all of trains are hauled by single-locomotive, Fig. 3 is the train schedule.

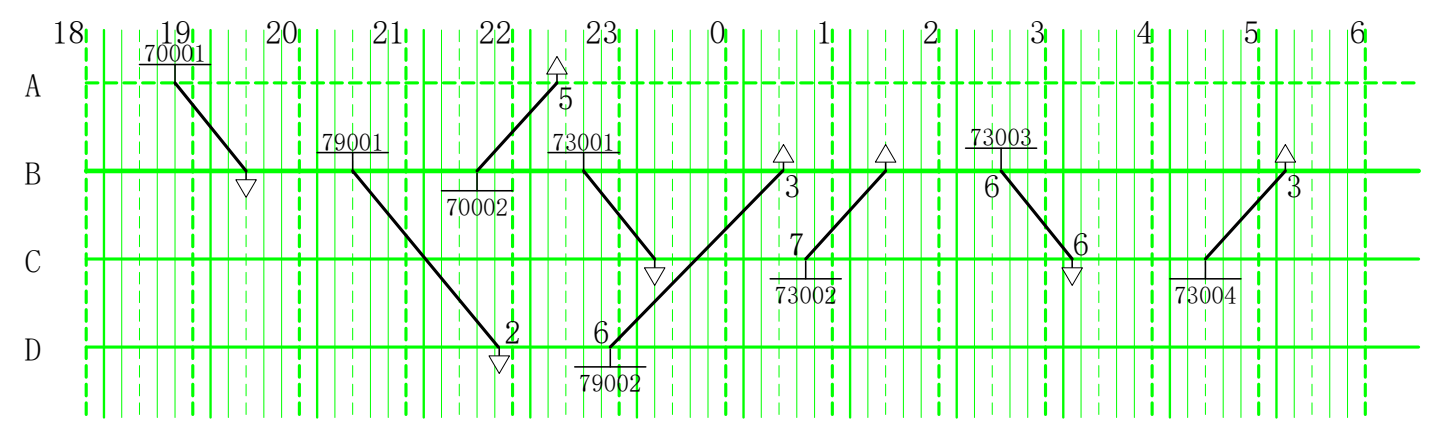

Fig. 3. Train schedule in section A-D

And the potential locomotive schedule in section A-D which is shown Fig. 4 in will be obtained, where a dotted line represents a locomotive. Fig. 4 shows that after hauling the train whose train number is 70001 to arrive at station B, the locomotive can haul the train whose train number is 70002 , 79001 or 73001 . When there are many kinds of locomotive traction schemes, the principle to determine the final scheme is the minimum detention time of locomotives at stations. 


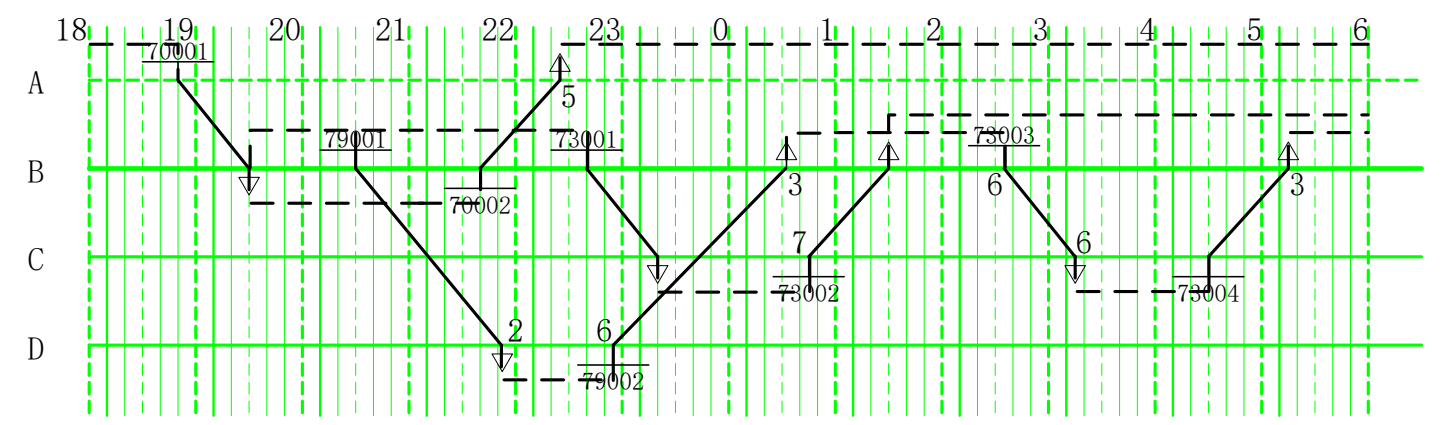

Fig. 4. Potential locomotive schedule in section A-D

This basic data needs to be processed as follows:

(1) Stations coding

For all stations in section A-D, it can code stations from any end of the railway and it starts with station A.

(2) Trains coding

The trains in the section A-D are sole, but due to the long and large train number, it is necessary for each train to code another sole and simple number.

(3) Time simplification

For the departure time and arrival time of each train, starting at the initial time (18:00) of the train schedule, time of $24 \mathrm{~h}$ can be converted to minutes, and for example, 19:33 can be converted to the $93^{\text {th }}$ minute.

(4) Generating coefficient matrix

According to Eq. (4), the coefficient matrix $c_{i, j}^{k}$ of the detention time of locomotives at stations will be generated by using C\# Program.

Result

By using MATLAB which includes Hungarian algorithm locomotive schedule is obtained. The results are illustrated as follow:

The minimum waiting time of locomotives at stations is

$$
T_{w}=Z=\sum_{k=1}^{4} \sum_{i=1}^{4} \sum_{j=1}^{4} c_{i, j}^{k} x_{i, j}^{k}=65+1175+37+1085+24+792+14+28=3220 \quad \text { (min). }
$$

The minimum standard detention time of locomotives at stations is

$$
T_{K}=125 \times 4+50 \times 4=700 \quad(\mathrm{~min}) .
$$

The running time of locomotives is

$$
T_{t}=40+45+40+43+40+43+82+67=400 \quad(\mathrm{~min}) .
$$

The minimum number of locomotives is

$$
M=\frac{T_{t}+T}{1440}=\frac{T_{t}+T_{K}+T_{w}}{1440}=\frac{400+700+3220}{1440}=\frac{4320}{1440}=3 .
$$

The coefficient of locomotive requirement is

$$
K_{d}=\frac{M}{n}=\frac{3}{8 / 2}=0.75 .
$$

The locomotive schedule can be expressed as an intuitive diagram, which is shown in Fig. 5 (dotted lines represent locomotives and solid lines represent trains). 


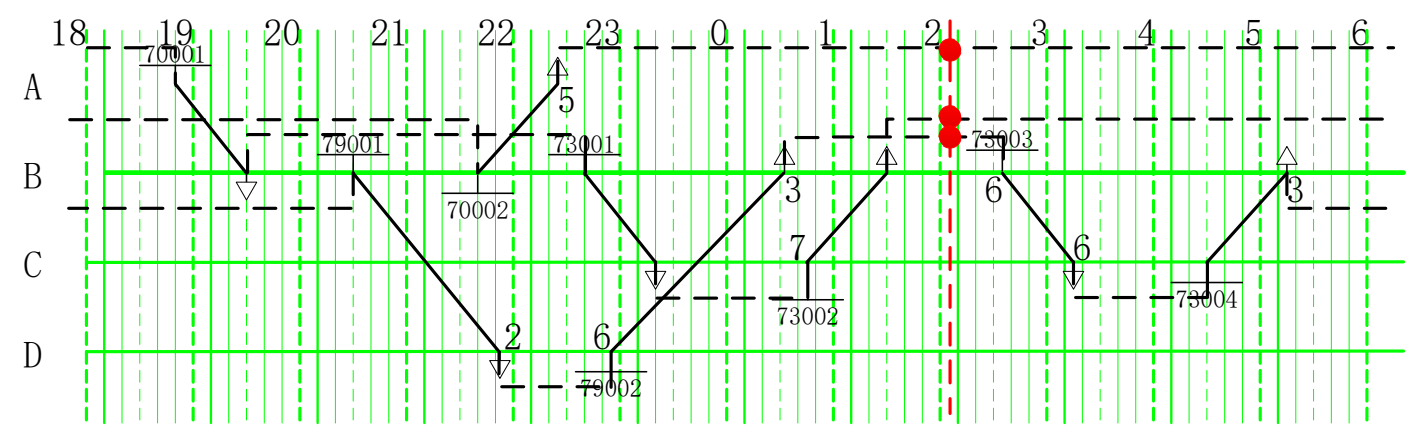

Fig. 5. Locomotive schedule in section A-D

Fig. 5 shows that there are 3 traction locomotives, which are equal to the calculation result, to haul trains.

Result analysis

(1) Analysis on the optimization of locomotive schedule

Comparing Fig. 4 with Fig. 5 it shows that, if we only consider the detention time of locomotives at the stations is longer than the standard time, there may be a variety of train connections. So, the number of available locomotives and coefficient of locomotive requirement are uncertain. Through overall consideration, the optimization model of heave-loaded locomotive schedule is established and then it can be solved by using Hungarian algorithm. It can not only make sure the uniqueness of locomotive traction tasks, but also complete established traction tasks with a minimum number of locomotives.

(2) Analysis on the equilibrium of locomotive schedule

The equilibrium degree of locomotive schedule of Fig. 5 can be obtained by using Eq. (9), which is

$$
\begin{aligned}
D= & \frac{1}{8} \sum_{k=1}^{4} \sum_{i=1}^{8} \sum_{j=1}^{8}\left(c_{i, j}^{k} x_{i, j}^{k}-\frac{Z}{8}\right)^{2} \\
= & \frac{1}{8} \times\left[\left(65-\frac{3220}{8}\right)^{2}+\left(1175-\frac{3220}{8}\right)^{2}+\left(37-\frac{3220}{8}\right)^{2}+\left(1085-\frac{3220}{8}\right)^{2}+\right. \\
& \left.\quad\left(24-\frac{3220}{8}\right)^{2}+\left(792-\frac{3220}{8}\right)^{2}+\left(14-\frac{3220}{8}\right)^{2}+\left(28-\frac{3220}{8}\right)^{2}\right] \\
= & 237027
\end{aligned}
$$

It shows that the equilibrium degree of locomotive schedule is large. The main reason is that there are no trains from 6:00 to 18:00 on the railway. Therefore, the diffidence between the detention time of locomotives at stations from 18:00 to 6:00 and the detention time of locomotives at stations from 6:00 to 18:00 is relatively large. If there are trains from 6:00 to 18:00, the equilibrium degree of locomotive schedule will be reduced and the locomotive schedule will be more balanced.

Analysis of Locomotive Schedule for 20kt Combined Trains on Da-qin Railway. In above section, it proves that the model of locomotive schedule is feasible. Then, the effectiveness of the proposed the model is needed to be validated which is conducted in this section.

\section{Line simplification}

Da-qin Railway has 26 stations and the line length is $653 \mathrm{~km}$; Qian-cao Railway has 5 stations and the line length is $137 \mathrm{~km}$. Jingtang Port-Donggang Railway Line has 5 stations and the line length is $45 \mathrm{~km}$. Some intermediate stations on the railway don't have the technical operation, so they are not displayed. And only the stations where locomotive depot or turnaround depot is located are considered. The simplified network of Daqin-Qiancao-Jingtang Port-Donggang Railway Line is shown in Fig. 6. 


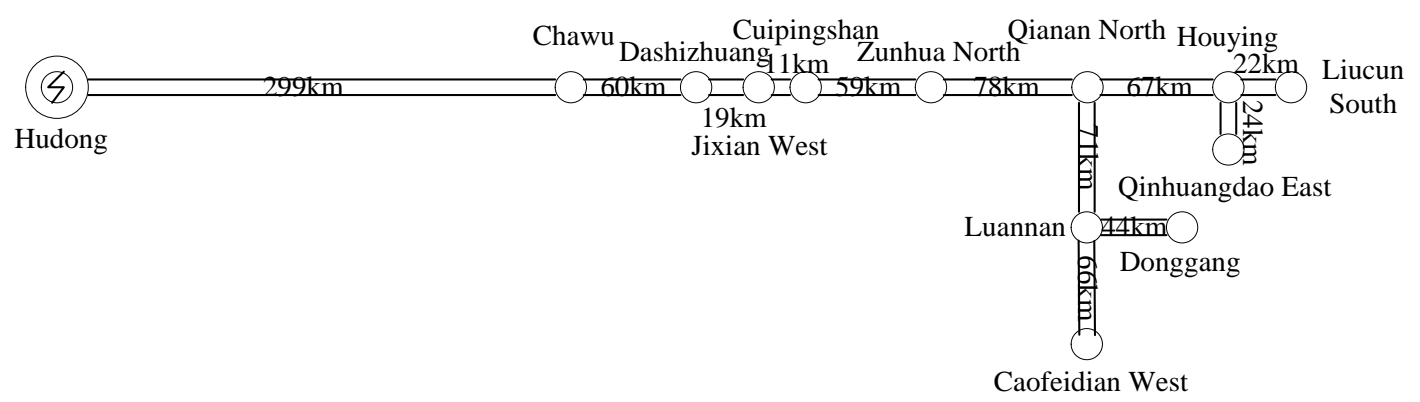

Fig. 6. Simplified network of Da-qin Railway

Hudong station is the station where locomotive depot is located, and the standard detention time of locomotives at stations is 250min. Liucun South station, Donggang station, Caofeidian West station and Qinhuangdao East station are the stations where locomotive turnaround depots are located. The standard detention time of locomotives at stations for Liucun South station is 220min. The standard detention time of locomotives at stations for Donggang station and Caofeidian West station is 100min. The standard detention time of locomotives at stations for Qinhuangdao East station is $125 \mathrm{~min}$. The minimum standard detention time of the other stations where the locomotives turn back is 50min.

There were 100 heavy-loaded trains in the heavy-load direction and 104 empty-wagon trains in the reverse direction on December 10, 2014.

Data processing

In reference to the method above-mentioned, then complete the data processing. And for the HXD locomotives, there are 328 trains which are hauled by single-locomotive after conversion, so the order of the coefficient matrix is 328 . For the SS4 locomotives, there are 56 trains which are hauled double-locomotive after conversion, so the order of the coefficient matrix is 56. In this paper, C\# Program is used to deal with such a huge data.

Result

The results are listed below:

The minimum waiting time of locomotives at stations are respectively

$$
\begin{aligned}
Z_{H X D} & =\sum_{k=1}^{13} \sum_{i=1}^{328} \sum_{j=1}^{328} c_{i, j}^{k} x_{i, j}^{k}=37116 \quad(\text { min }), \\
Z_{S S 4} & =\sum_{k=1}^{13} \sum_{i=1}^{56} \sum_{j=1}^{56} c_{i, j}^{k} x_{i, j}^{k}=10409 \quad(\text { min }) .
\end{aligned}
$$

The minimum standard detention time of locomotives at stations are respectively

$$
\begin{gathered}
T_{K}^{H X D}=164 \times 250+76 \times 220+14 \times 125+44 \times 100+30 \times 50=65370 \quad(\mathrm{~min}), \\
T_{K}^{S S 4}=28 \times 250+28 \times 50=8400 \quad(\mathrm{~min}) .
\end{gathered}
$$

The running time of locomotives are respectively

$$
\begin{gathered}
T_{t}^{H X D}=198474 \quad(\min ), \\
T_{t}^{S S 4}=24391 \quad(\min ) .
\end{gathered}
$$

According to that data, the minimum number of locomotives are respectively

$$
\begin{aligned}
& M_{H X D}=\frac{T_{t}+T}{1440}=\frac{T_{t}+T_{K}+Z}{1440}=\frac{198474+65370+37116}{1440}=\frac{300960}{1440}=209, \\
& M_{S S 4}=\frac{T_{t}+T}{1440}=\frac{T_{t}+T_{K}+Z}{1440}=\frac{24391+8400+10409}{1440} \times 2=\frac{43200}{1440} \times 2=60 .
\end{aligned}
$$

So, the coefficient of locomotive requirement are respectively

$$
\begin{gathered}
K_{d}^{H X D}=\frac{M_{H X D}}{n}=\frac{209}{160 / 2}=2.61, \\
K_{d}^{S S 4}=\frac{M_{S S 4}}{n}=\frac{30 \times 2}{56 / 2}=2.14 .
\end{gathered}
$$


According to the best connection matrix obtained, locomotive connection scheme for $20 \mathrm{kt}$ combined trains on Da-qin Railway can be obtained. The locomotive connection scheme of SS4 locomotives are as follows:

H16012 $\rightarrow$ 52(single-locomotive) $\rightarrow$ H16016 $\rightarrow$ 54(single-locomotive) $\rightarrow$ H16004

$\rightarrow \mathrm{H} 19609 \rightarrow \mathrm{H} 19504 \rightarrow \mathrm{H} 19605 \rightarrow{ }_{\mathrm{H} 19512} \rightarrow$ 53(single-locomotive) $\rightarrow$ H16018

$\rightarrow$ 56 (single-locomotive) $\rightarrow$ H16002 $\rightarrow$ 48( single-locomotive) $\rightarrow$ H15506

$\rightarrow$ 47(single-locomotive) $\rightarrow \mathrm{H} 16010 \rightarrow 19601 \rightarrow \mathrm{H} 19508 \rightarrow$ 46(single-locomotive)

$\rightarrow \mathrm{H} 16008 \rightarrow \mathrm{H} 15501 \rightarrow \mathrm{H} 15504 \rightarrow \mathrm{H} 19607 \rightarrow \mathrm{H} 19516 \rightarrow 19615 \rightarrow \mathrm{H} 19514 \rightarrow{ }_{\mathrm{H} 28501}$

$\rightarrow \mathrm{H} 28510 \rightarrow$ 51 (single-locomotive) $\rightarrow \mathrm{H} 16014 \rightarrow \mathrm{H} 19603 \rightarrow \mathrm{H} 19502 \rightarrow \mathrm{H} 28507 \rightarrow \mathrm{H} 28512$

$\rightarrow$ 49(attached locomotive) $\rightarrow \mathrm{H} 15508 \rightarrow \mathrm{H} 28503 \rightarrow \mathrm{H} 28508 \rightarrow$ 50(single-locomotive)

$\rightarrow \mathrm{H} 16012 \rightarrow \mathrm{H} 19611 \rightarrow \mathrm{H} 19506 \rightarrow \rightarrow_{\mathrm{H} 28511} \rightarrow \mathrm{H} 28502 \rightarrow \mathrm{H} 15503 \rightarrow \mathrm{H} 15502$

$\rightarrow$ 55(attached locomotive) $\rightarrow \mathrm{H} 15510 \rightarrow{ }_{\mathrm{H} 28505} \rightarrow \mathrm{H} 28506 \rightarrow$ 45(single-locomotive)

$\rightarrow \mathrm{H} 16006 \rightarrow \mathrm{H} 19611$

$\mathrm{H} 19613 \rightarrow \mathrm{H} 19510 \rightarrow \mathrm{H} 19613$

$\mathrm{H} 28504 \rightarrow \mathrm{H} 28509 \rightarrow \mathrm{H} 28504$.

Analysis of result

(1) Analyze the number of available locomotives

The number of available (the total number of locomotives after deducting locomotives in maintenance and preparation) on Da-qin Railway on December 10, 2014 is shown in

\begin{tabular}{cccc}
\hline $\begin{array}{c}\text { Types of } \\
\text { locomotive }\end{array}$ & $\begin{array}{c}\text { Daily number of } \\
\text { locomotives }\end{array}$ & $\begin{array}{c}\text { Types of } \\
\text { locomotive }\end{array}$ & $\begin{array}{c}\text { Daily number of } \\
\text { locomotives }\end{array}$ \\
\hline HXD1 & 130 & SS4 & 109 \\
HXD2 & 92 & - & - \\
\hline
\end{tabular}

Table 2. The daily number of locomotives on Da-qin Railway on December 10, 2014 [19]

It is obvious that the locomotive schedule obtained by using the model can save the number of HXD locomotives: $130+92-209=13$ and the number of SS4 locomotives: $109-30 \times 2=49$. Therefore, if only the minimum number of locomotives is considered, the optimization model of heavy-loaded locomotive schedule is quite optimized.

(2) Analyze on the equilibrium of locomotive schedule

The equilibrium degree of locomotive schedule of HXD locomotives and SS4 locomotives are respectively

$$
\begin{aligned}
D_{H X D} & =\frac{1}{328} \sum_{k=1}^{13} \sum_{i=1}^{328} \sum_{j=1}^{328}\left(c_{i, j}^{k} x_{i, j}^{k}-\frac{Z}{328}\right)^{2}=52379, \\
D_{S S 4} & =\frac{1}{56} \sum_{k=1}^{13} \sum_{i=1}^{56} \sum_{j=1}^{56}\left(c_{i, j}^{k} x_{i, j}^{k}-\frac{Z}{56}\right)^{2}=74044 .
\end{aligned}
$$

The equilibrium degree of the locomotive schedule for HXD locomotives and SS4 locomotives are smaller than the equilibrium degree of that case, which shows that this locomotive schedule is more balanced. And the equilibrium degree of SS4 locomotives is larger than the equilibrium degree of HXD locomotives, which shows that the schedule of HXD locomotives is more balance than SS4 locomotives. The large equilibrium degree of SS4 locomotives may be due to the small detention time of locomotives at stations.

Research and Analysis of Locomotive Schedule Optimization for 30kt Combined Trains on Da-qin Railway. On the basis of the potential train schedule for 30kt combined trains which is given by "Research on Capacity Coordination of Station and Section on Heavy-Haul Railway", the practical and feasible locomotive connection scheme can be determined by using the optimization model.

In reference to the method above-mentioned, then complete the data processing. 
Result

The minimum waiting time of locomotives at stations are respectively

$$
\begin{aligned}
Z_{H X D} & =\sum_{k=1}^{13} \sum_{i=1}^{406} \sum_{j=1}^{406} c_{i, j}^{k} x_{i, j}^{k}=44838 \quad(\text { min }), \\
Z_{S S 4} & =\sum_{k=1}^{13} \sum_{i=1}^{52} \sum_{j=1}^{52} c_{i, j}^{k} x_{i, j}^{k}=10314 \quad(\text { min }) .
\end{aligned}
$$

The minimum standard detention time of locomotives at stations are respectively

$$
\begin{gathered}
T_{K}^{H X D}=203 \times 250+64 \times 220+65 \times 100+74 \times 50=75030 \quad(\text { min }), \\
T_{K}^{S S 4}=26 \times 250+26 \times 50=7800 \quad(\text { min }) .
\end{gathered}
$$

The running time of locomotives are respectively

$$
\begin{aligned}
T_{t}^{H X D} & =241572 \quad(\mathrm{~min}), \\
T_{t}^{S S 4} & =23646 \quad(\mathrm{~min}) .
\end{aligned}
$$

According to that data, the minimum number of locomotives are respectively

$$
\begin{gathered}
M_{H X D}=\frac{T_{t}+T}{1440}=\frac{T_{t}+T_{K}+Z}{1440}=\frac{241572+75030+44838}{1440}=\frac{361440}{1440}=251, \\
M_{S S 4}=\frac{T_{t}+T}{1440}=\frac{T_{t}+T_{K}+Z}{1440}=\frac{23646+7800+10314}{1440}=\frac{41760}{1440}=29 .
\end{gathered}
$$

So, the coefficient of locomotive requirement are respectively

$$
\begin{gathered}
K_{d}^{H X D}=\frac{M_{H X D}}{n}=\frac{251}{59+3+20}=3.06, \\
K_{d}^{S S 4}=\frac{M_{S S 4}}{n}=\frac{29 \times 2}{52 / 2}=2.23 .
\end{gathered}
$$

The locomotive connection scheme and the locomotive schedule

According to the best connection matrix obtained, locomotive connection scheme for $30 \mathrm{kt}$ combined train on Da-qin Railway can be obtained. The locomotive connection scheme of SS4 locomotives are as follows:

$$
\begin{aligned}
& \mathrm{H} 16004 \rightarrow \mathrm{H} 28503 \rightarrow \mathrm{H} 28508 \rightarrow \mathrm{H} 17215 \rightarrow \mathrm{H} 17208 \rightarrow \mathrm{H} 17203 \rightarrow \mathrm{H} 17214 \rightarrow{ }_{\mathrm{H} 28507} \\
& \rightarrow \mathrm{H} 28512 \rightarrow \mathrm{H} 28501 \rightarrow{ }_{\mathrm{H} 28506} \rightarrow \mathrm{H} 28515 \rightarrow \mathrm{H} 28504 \rightarrow \rightarrow_{\mathrm{H} 15505} \rightarrow \mathrm{H} 15502 \rightarrow \mathrm{H} 19605 \\
& \rightarrow \mathrm{H} 19502 \rightarrow \mathrm{H} 17209 \rightarrow \mathrm{H} 17202 \rightarrow \mathrm{H} 17211 \rightarrow \mathrm{H} 17206 \rightarrow \rightarrow_{\mathrm{H} 17213} \rightarrow \mathrm{H} 17210 \rightarrow{ }_{\mathrm{H} 15503} \\
& \rightarrow \mathrm{H} 15506 \rightarrow \mathrm{H} 28505 \rightarrow \mathrm{H} 28510 \rightarrow \mathrm{H} 17201 \rightarrow \mathrm{H} 17212 \rightarrow \mathrm{H} 17205 \rightarrow \mathrm{H} 17216 \rightarrow{ }_{\mathrm{H} 28509} \\
& \rightarrow \mathrm{H} 28514 \rightarrow \mathrm{H} 16003 \rightarrow \mathrm{H} 16008 \rightarrow \mathrm{H} 28511 \rightarrow \mathrm{H} 28516 \rightarrow{ }_{\mathrm{H} 17207} \rightarrow \mathrm{H} 17204 \rightarrow{ }_{\mathrm{H} 28513} \\
& \rightarrow \mathrm{H} 28502 \rightarrow \mathrm{H} 16005 \rightarrow \mathrm{H} 16002 \rightarrow \mathrm{H} 16007 \rightarrow \mathrm{H} 16004 \\
& \mathrm{H} 19601 \rightarrow \mathrm{H} 19504 \rightarrow \mathrm{H} 15501 \rightarrow \rightarrow_{\mathrm{H} 15504} \rightarrow \mathrm{H} 16001 \rightarrow \rightarrow_{\mathrm{H} 16006} \rightarrow \mathrm{H} 19603 \rightarrow{ }_{\mathrm{H} 19506}
\end{aligned}
$$

$\rightarrow$ H19601.

The locomotive connection scheme can be shown to be a locomotives working diagram which is shown in Fig. 7. 


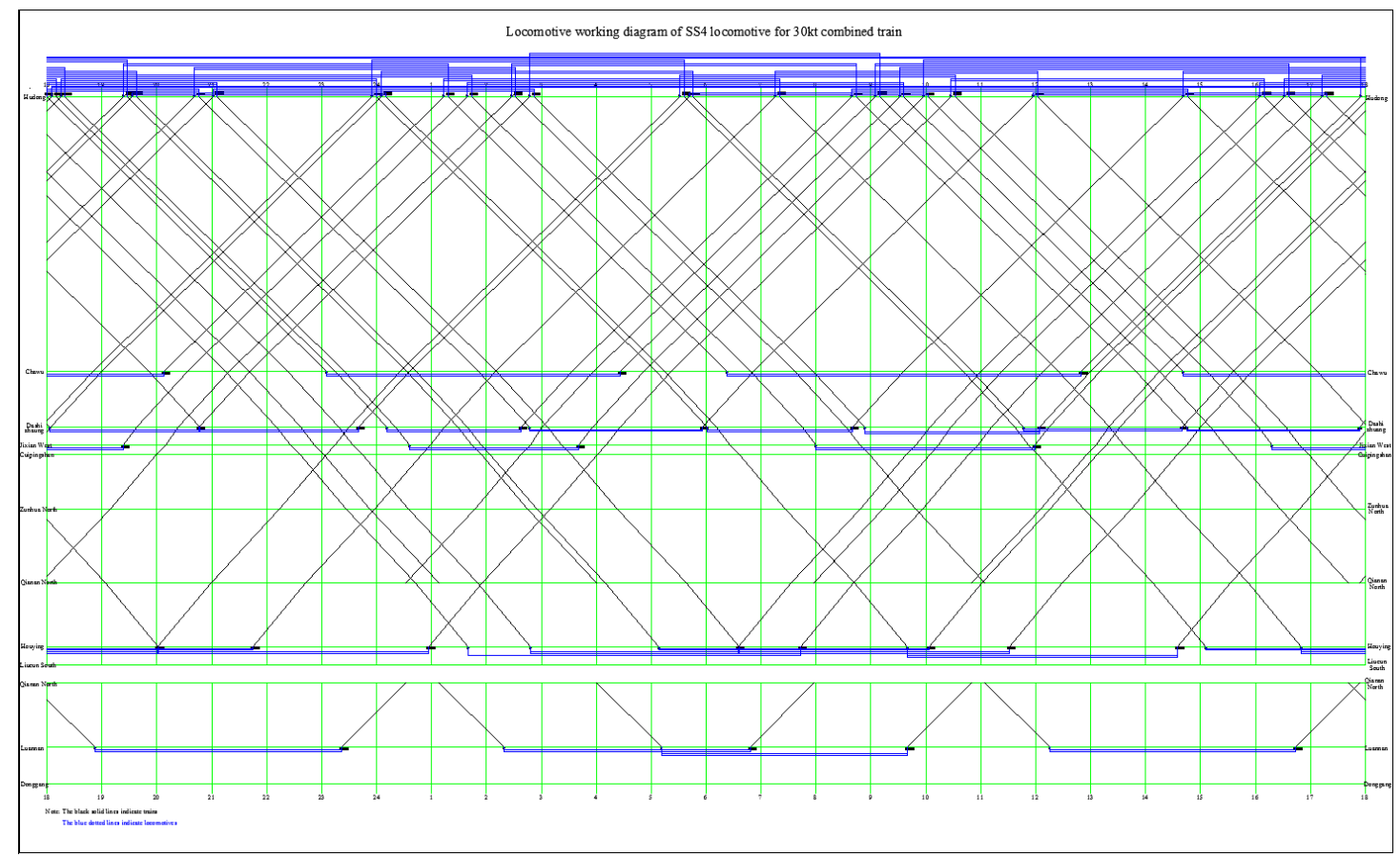

Fig. 7. Locomotive working diagram of SS4 locomotives for 30kt combined trains Analysis of Result

(1) Analyze the number of available locomotives

The daily average number of available locomotives in 2014 can be treated as the daily number of locomotives which is shown in Table 3.

\begin{tabular}{cccc}
\hline $\begin{array}{c}\text { Types of } \\
\text { locomotive }\end{array}$ & $\begin{array}{c}\text { The possessive } \\
\text { quantity of } \\
\text { locomotives }\end{array}$ & $\begin{array}{c}\text { The locomotive } \\
\text { number of 20kt } \\
\text { combined trains }\end{array}$ & $\begin{array}{c}\text { The locomotive number } \\
\text { of 30kt combined trains }\end{array}$ \\
\hline HXD & 251 & 209 & 251 \\
SS4 & 105 & 60 & 58 \\
\hline
\end{tabular}

Table 3. The average number of locomotives in Da-qin Railway in 2014

Analyzing the data in Table 3, it is obvious that the locomotive schedule just meets the needs of HXD locomotives and saves the number of SS4 locomotives: $105-29 \times 2=47$. Therefore, for the given train schedule for $30 \mathrm{kt}$ combined trains, all of trains will be operated smoothly.

(2) Analyze the equilibrium of locomotive schedule

The equilibrium degree of locomotive schedule of HXD locomotives and SS4 locomotives are respectively

$$
\begin{gathered}
D_{H X D}=\frac{1}{406} \sum_{k=1}^{13} \sum_{i=1}^{406} \sum_{j=1}^{406}\left(c_{i, j}^{k} x_{i, j}^{k}-\frac{Z}{406}\right)^{2}=40812, \\
D_{S S 4}=\frac{1}{52} \sum_{k=1}^{13} \sum_{i=1}^{52} \sum_{j=1}^{52}\left(c_{i, j}^{k} x_{i, j}^{k}-\frac{Z}{52}\right)^{2}=47201 .
\end{gathered}
$$

Compared the potential locomotive schedule for 30kt combined trains with the optimized locomotive schedule of the current train schedule, the equilibrium degree of the locomotive schedule of HXD locomotives and SS4 locomotives for 30kt combined trains are smaller than the equilibrium degree of $20 \mathrm{kt}$ combined trains. It shows that the potential locomotive schedule is more balanced. And the equilibrium degree of SS4 locomotives is larger than the equilibrium degree of HXD locomotives, which shows that the schedule of HXD locomotives is more balanced than SS4 locomotives. 


\section{Conclusions}

Improving the efficiency, reliability and balance of locomotive schedule is one of the important tasks of improving transport organization and management. The rationality of locomotive schedule has a direct impact on the transportation efficiency and benefit. Therefore, it is necessary to research how to improve the locomotive scheduling efficiency.

Comparing the locomotive schedule for 30kt combined trains of the potential train schedule with the optimized locomotive schedule for 20kt combined trains of the current train schedule, the following conclusions can be drawn:

1) For the given feasible train schedule for 30kt combined trains on Da-qin Railway, all of trains will be operated smoothly. And the HXD locomotives just meet the needs while the remaining number of SS4 locomotives is 44. Therefore, in the actual transport organization process, it needs to strengthen monitoring and maintenance of HXD locomotives; and for SS4 locomotives. It is obvious an effective way that increases the quantities of locomotives for operating trains to reduce the equilibrium degree of locomotive schedule.

2) Increase the standard detention time of locomotives at stations can reduce the equilibrium degree of the locomotive schedule and make locomotive schedule more balance.

3) Strengthen interconnection research between different locomotive types. At present, 15kt combined trains are hauled by two HXD locomotives. The hauling weight of one HXD locomotive is 10 kilotons, so there will be some waste of hauling weight. The hauling weight of one SS4 locomotive is 5 kilotons. Therefore, if the reconnection operation and synchronization control between HXD locomotives. SS4 locomotives can be realized by LOCOTROL technology, one 15kt combined train will be successfully hauled by one HXD locomotive and one SS4 locomotive, which will release the hauling weight and make the transportation tasks complete more smoothly.

\section{Acknowledgements}

The material in this paper is based on research supported by "the Fundamental Research Funds for the Central Universities" under grant No.2015JBM057 "Research on Assessment and Improvement Train Schedule for High Speed Railway Based on Multi-Resource Constraint" and China Railway Corporation under grant No. 2014X001-B "Study on Transportation Scheduling for 30kt Combined Train in Da-qin Railway". And the real data in this paper is based on research supported by TaiYuan RailWay Administration.

\section{References}

[1] National Bureau of Statistics of the People's Republic of China. China Statistical Yearbook [M]. China Statistics Press, 2006-2015. (In Chinese)

[2] Z. H. Cheng, et al. Application of Reliability Centered Maintenance in Railway Locomotive-a Case Study [C]// Proceedings of the First International Conference on Maintenance Engineering, pp.269-275, 2006.

[3] F. Y. Zheng and Q. C. Wei. Heavy-Axle Load Mechanical Effects on Railway Tracks [J]. New Technologies of Railway Engineering, pp.233-236, 2012.

[4] W. H. Chang. Research on the Mechanical Properties of 30t Axle Heavy-Loaded Railway Track Structure [D]. Beijing Jiaotong University, 2009. (In Chinese)

[5] X. Mu, et al. Enlightenment of Foreign Railway Heavy-Loaded Transport to the Development of China's Railway Freight Transport [J]. Railway Economics Research, 2013. (In Chinese)

[6] T. Raviv and M. Kaspi. The Locomotive Fleet Fueling Problem [J]. Operations Research Letters, vol.40, pp.39-45, 2012.

[7] V. P. Kumar and M. Bierlaire. Optimizing Fueling Decisions for Locomotives in Railroad Networks [J]. General Information, vol.49, pp.149-159, 2011. 
[8] B. Ji, et al. Study on Intercommunication between Locomotives for Heavy Haul Train on Da-qin Line [J]. Railway Locomotive \& Car, vol.30, pp.12-14, 2010. (In Chinese)

[9] D. Ruppert, et al. Root Cause Analysis Increases Locomotive Reliability at AMTRAK [C]//

Proceedings of the ASME/IEEE Joint Rail Conference and the ASME Internal Combustion Engine

Division Spring Technical Conference, pp.305-310, 2007.

[10] C. C. Kuo and G. A. Nicholls. A Mathematical Modeling Approach to Improving Locomotive Utilization at a Freight Railroad [J]. Omega - International Journal of Management Science, vol.35, pp.472-485, 2007.

[11] M. Aronsson, P. Kreuger and J. Gjerdrum. An Efficient MIP Model for Locomotive Routing and Scheduling [C]// 12th International Conference on Computer System Design and Operation in Railways and Other Transit Systems, vol.114, pp.963-973, 2010.

[12] B. Vaidyanathan, R. K. Ahuja and J. B. Orlin. The Locomotive Routing Problem [J]. Transportation Science, vol.42, pp.492-507, 2008.

[13] K. Ghoseiri and S. F. Ghannadpour. A Hybrid Genetic Algorithm for Multi-Depot Homogenous Locomotive Assignment with Time Windows [J]. Applied Soft Computing, vol.10, pp.53-65, 2010. [14] S. Kasalica, D. Mandic and V. Vukadinovic. Locomotive Assignment Optimization Including Train Delays [J]. Promet-Traffic \& Transportation, vol.25, pp.421-429, 2013.

[15] S. Rouillon, G. Desaulniers and F. Soumis. An Extended Branch-and-Bound Method for Locomotive Assignment [J]. Transportation Research Part B Methodological, vol.40, pp.404-423, 2006.

[16] D. Teichmann, et al. Locomotive Assignment Problem with Heterogeneous Vehicle Fleet and Hiring External Locomotives [J]. Mathematical Problems in Engineering, 2015.

[17] C. B. Li, et al. An Improved Ant Colony Algorithm for Making Locomotive Working Diagram $[C] / / 2^{\text {nd }}$ International Conference on Modelling and Simulation, pp.63-68, 2009.

[18] H. Yang. Railway Transport Organization ( $3^{\text {rd }}$ Edition) [M]. China Railway Publishing House, 2006. (In Chinese)

[19] TaiYuan RailWay Administration. Technical Information of Train Schedule [S]. TaiYuan RailWay Administration, 2012. (In Chinese) 